\title{
Intermedin improves cardiac function and sympathetic neural remodeling in a rat model of post myocardial infarction heart failure
}

\author{
BIN XU, HAO XU, HENG CAO, XIAOXIAO LIU, CHUNHUAN QIN, YANZHOU ZHAO, \\ XIAOLIN HAN and HONGLI LI \\ Department of Cardiology, Shanghai General Hospital, Shanghai Jiao Tong University School of Medicine, \\ Shanghai 200080, P.R. China
}

Received May 11, 2016; Accepted March 27, 2017

DOI: $10.3892 / \mathrm{mmr} .2017 .6776$

\begin{abstract}
Emerging evidence has suggested that intermedin (IMD), a novel member of the calcitonin gene-related peptide (CGRP) family, has a wide range of cardioprotective effects. The present study investigated the effects of long-term administration of IMD on cardiac function and sympathetic neural remodeling in heart failure (HF) rats, and studied potential underlying mechanism. HF was induced in rats by myocardial infarction (MI). Male Sprague Dawley rats were randomly assigned to either saline or IMD $(0.6 \mu \mathrm{g} / \mathrm{kg} / \mathrm{h})$ treatment groups for 4 weeks post-MI. Another group of sham-operated rats served as controls. Cardiac function was assessed by echocardiography, cardiac catheterization and plasma level of B-type natriuretic peptide (BNP). Cardiac sympathetic neural remodeling was assessed by immunohistochemistical study of tyrosine hydroxylase (TH) and growth associated protein 43 (GAP43) immunoreactive nerve fibers. The protein expression levels of nerve growth factor (NGF), TH and GAP43 in the ventricular myocardium were studied by western blotting. Ventricular fibrillation threshold (VFT) was determined to evaluate the incidence of ventricular arrhythmia. Oxidative stress was assessed by detecting the activity of superoxide dismutase and the level of malondialdehyde. Compared with rats administrated with saline, IMD significantly improved cardiac function, decreased the plasma BNP level, attenuated sympathetic neural remodeling, increased VFT and suppressed oxidative stress. In conclusion, these results indicated that IMD
\end{abstract}

Correspondence to: Dr Xiaolin Han or Dr Hongli Li, Department of Cardiology, Shanghai General Hospital, Shanghai Jiao Tong University School of Medicine, 100 Haining Road, Shanghai 200080, P.R. China

E-mail: 13371989275@163.com

E-mail: drsyhonglili@126.com

Key words: intermedin, cardiac function, sympathetic neural remodeling, ventricular arrhythmia, oxidative stress, heart failure prevents ventricle remodeling and improves the performance of a failing heart. In addition, IMD attenuated sympathetic neural remodeling and reduced the incidence of ventricular arrhythmia, which may contribute to its anti-oxidative property. These results implicate IMD as a potential therapeutic agent for the treatment of HF.

\section{Introduction}

Currently, ischemic heart disease is the primary cause of heart failure (HF) (1). Ventricle remodeling and cardiac sympathetic hyperactivity following myocardial infarction (MI) contribute to the development of HF $(2,3)$. Increased cardiac sympathetic nerve activity causes sympathetic hyperinnervation and heterogeneous nerve sprouting, also known as sympathetic neural remodeling, which causes ventricular arrhythmia and sudden cardiac death (SCD) $(4,5)$. Oxidative stress has been demonstrated to serve an important role in sympathetic innervation following MI, which promotes sympathetic neural remodeling and arrhythmia via increasing the expression of nerve growth factor (NGF) (6). Anti-oxidative stress therapy significantly decreases the density of sympathetic nerve and the protein expression of NGF following MI (7-9).

Intermedin (IMD) is a novel member of the calcitonin gene-related peptide (CGRP) family, signaling via calcitonin receptor-like receptor/receptor activity modifying protein (CRLR/RAMP) complexes (10). It was reported that IMD has beneficial effects on the cardiovascular system (11). The expression of IMD is increased in the failing heart and may have a certain pathophysiological role in HF (12). A subsequent study demonstrated its favorable haemodynamic, hormonal and renal actions in a sheep model of experimental HF (13). Furthermore, IMD protects against myocardial and renal ischemia/reperfusion injury via inhibition of oxidative stress in animal models (14-16). However, whether IMD may ameliorate sympathetic neural remodeling via anti-oxidative effects following MI remains unclear.

The present study investigated the effects of long-term administration of exogenous IMD on cardiac function and sympathetic neural remodeling in a rat model of post-MI HF. 


\section{Materials and methods}

Peptide synthesis. Human IMD (IMD1-53) with the sequence His-Ser-Gly-Pro-Arg-Arg-Thr-Gln-Ala-Gln-Leu-Leu-Arg-Val -Gly-Cys-Val-Leu-Gly-Thr-Cys-Gln-Val-Gln-Asn-Leu-Ser-Hi s-Arg-Leu-Trp-Gln-Leu-Met-Gly-Pro-Ala-Gly-Arg-Gln-AspSer-Ala-Pro-Val-Asp-Pro-Ser-Ser-Pro-His-Ser-Tyr-NH2 with an intramolecular disulfide bond between Cys16-Cys21 (17) was synthesized by ShineGene Bio-Technologies (Shanghai, China).

Establishment of animal models. Adult male Sprague Dawley rats, (weight, 280-320 g; n=60), were supplied by Sino-British Sippr/BK Lab Animal Ltd. (Shanghai, China) The animal experiment was in compliance with the National Research Council's protocol for the Care and Use of Laboratory Animals, and was approved by the Animal Care Committee of Shanghai General Hospital (Shanghai, China). The HF model was induced in Sprague Dawley rats by ligation of the left anterior descending (LAD) coronary artery. Briefly, all rats were anesthetized with intraperitoneal injection of $1 \%$ sodium pentobarbital (40 mg/kg; Sinopharm Chemical Reagent Co., Ltd., Shanghai, China), endotracheal intubated and mechanically ventilated with a small animal ventilator. Then all rats underwent thoracotomy and pericardiotomy, and the LAD coronary artery was ligated by a 6-0 prolene suture at the origin. Successful myocardium ischemia was verified by ST-segment elevation on an electrocardiogram. The sham group rats underwent same procedure without LAD coronary artery ligation.

Animal grouping and treatment. The rats that survived $24 \mathrm{~h}$ after surgery were randomly assigned to the following 3 groups: Sham $(\mathrm{n}=10)$, where rats were administrated subcutaneously with saline $(0.6 \mu \mathrm{g} / \mathrm{kg} / \mathrm{h})$ by a mini-osmotic pump (Alzet model 2004; DURECT Corporation, Cupertino, CA, USA) for 4 weeks; HF ( $n=18)$, where rats were administrated subcutaneously with saline $(0.6 \mu \mathrm{g} / \mathrm{kg} / \mathrm{h})$ by a mini-osmotic pump for 4 weeks; and HF rats with IMD treatment (HF+IMD group; $n=20$ ), where rats were daily administrated subcutaneously with IMD $(0.6 \mu \mathrm{g} / \mathrm{kg} / \mathrm{h})(13)$ by a mini-osmotic pump for 4 weeks. After 4 weeks, rats underwent echocardiographic examination, haemodynamic measurement and ventricular fibrillation threshold (VFT) determination. After that, animals were sacrificed and hearts were excised for further study.

Echocardiography and haemodynamic measurement. Rats were lightly anesthetized with $1 \%$ sodium pentobarbital, (40 mg/kg; Sinopharm Chemical Reagent Co., Ltd.) and transthoracic echocardiography was performed with a $30 \mathrm{MHz}$ high frequency transducer (VisualSonics Vevo770; VisualSonics, Inc., Toronto, ON, Canada) as previously described (18). End-diastolic and end-systolic left ventricle diameters were measured by M-mode tracing. Left ventricular ejection fraction (LVEF) and fractional shortening (LVFS) were calculated. Following echocardiography study, haemodynamic parameters were measured using the BL-420E Biological system (Chengdu Tai-Meng Science and Technology Co., Ltd., Chengdu, Sichuan, China). Briefly, a catheter was inserted into the right common carotid artery to monitor the arterial blood pressure. Subsequently the catheter was inserted into the left ventricle to record the left ventricular end-diastolic pressure (LVEDP) and the maximal rate of left ventricular pressure increase and decrease $\left( \pm \mathrm{LVdp} / \mathrm{dt}_{\max }\right)$.

Determination of VFT. A total of 5 rats in the HF+IMD group and 7 rats in the HF group succumbed during the haemodynamic measurement and anesthesia prior to the second thoracotomy. Therefore, 10 rats in each group were selected for the determination of VFT. VFT measurement was performed as previously described (19). A bipolar needle pacing electrode was penetrated into the peri-infarct zone, which was defined as the zone with a $<3-\mathrm{mm}$ width located between the infarct zone and the non-infarct area. A train of rectangular $4 \mathrm{~ms}$ pacing pulses was performed for $5 \mathrm{sec}$ at a frequency of $50 \mathrm{HZ}$ using the BL-420E Biological system. The interval between pulse trains was $1 \mathrm{~min}$. The pacing voltage of the first pulse train started at $4 \mathrm{~V}$ and progressively augmented by a step of $1 \mathrm{~V}$. The VFT was the minimum voltage which induced ventricular fibrillation (sustained $>2 \mathrm{sec}$ ).

Determination of infarct size and tissue preparation. Following determination of VFT, all rats were sacrificed by injecting a fatal dose of pentobarbital $(200 \mathrm{mg} / \mathrm{kg}$; Sinopharm Chemical Reagent Co., Ltd.). The heart was excised and rinsed in cold saline. The atria and right ventricle were trimmed off, and the left ventricle was weighed. A transverse section obtained from the papillary muscle level of the left ventricle was fixed in $10 \%$ formalin and embedded in paraffin. The section was then sectioned into $4-\mu \mathrm{m}$ slices and stained with haematoxylin-eosin for infarct size measurement. The infarct size was determined as previously described (9). Rats with infarct size $<30 \%$ were excluded from analysis $(\mathrm{n}=2$ from the $\mathrm{HF}+\mathrm{IMD}$ group; $\mathrm{n}=1$ from the HF group) (20). Left ventricular tissues from the peri-infarct zone were fixed in $10 \%$ formalin for $24 \mathrm{~h}$ and embedded for immunohistochemical studies and frozen in liquid nitrogen for western blot analysis.

Immunohistochemical studies. Paraffin-embedded tissues were sectioned into $4-\mu \mathrm{m}$ slices using a microtome (Leica Microsystems, Wetzlar, Germany). Following deparaffinization and rehydration, slides were treated with $3 \% \mathrm{H}_{2} \mathrm{O}_{2}$ and subjected to heat mediated antigen retrieval with citrate buffer. The slides were incubated with primary antibodies against tyrosine hydroxylase (TH; 1:200, cat no. ab75875) and growth associated protein 43 (GAP43; 1:500, cat no. ab75810) (both from Abcam, Cambridge, UK) overnight at $4^{\circ} \mathrm{C}$. Following this, the slides were washed and incubated with a horseradish peroxidase (HRP)-conjugated immunoglobulin G secondary antibody (cat no. GK600705; Gene Tech Biological Technology, Inc., Shanghai, China) for $30 \mathrm{~min}$ at room temperature. The immunoreactivity was developed with 3, 3'-diaminobenzidine tetrahydrochloride (Biological Technology, Inc.). Slides were counterstained with hematoxylin and analyzed under a light microscope.

The densities of TH- and GAP43-positive nerve fibers were determined using Image-Pro Plus version 6.0 software (Media Cybernetics, Inc., Rockville, MD, USA) and expressed as the nerve area divided by the total area examined. For each heart, one slide was taken to study. Each slide was divided into four 
A

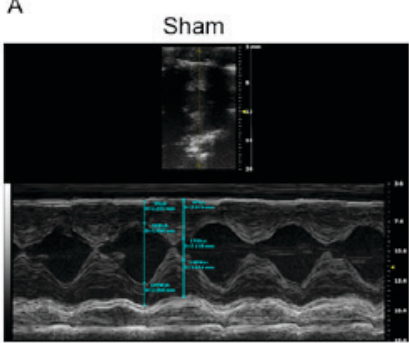

B

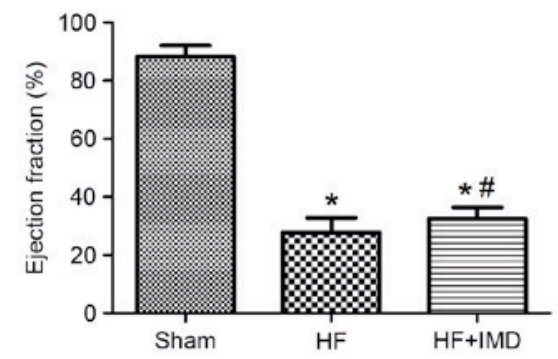

$\mathrm{HF}$
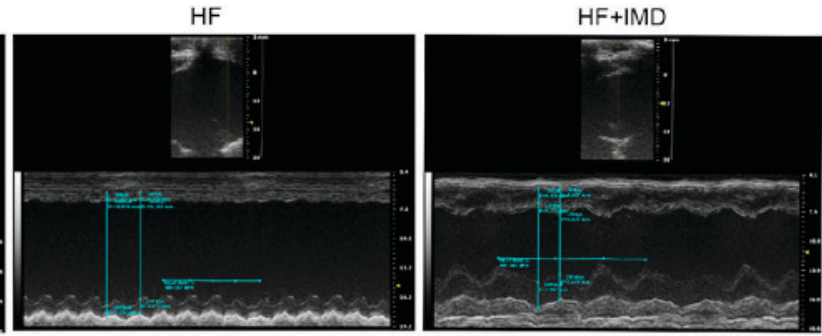

C

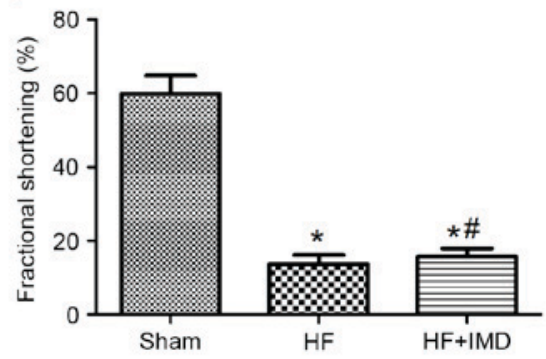

Figure 1. IMD improves cardiac function in rats with post-myocardial infarction HF. (A) Representative echocardiograms in the sham, HF and HF+IMD groups (B) Ejection fraction and (C) fractional shortening were also calculated and compared between the three groups. Data are presented as the mean \pm standard deviation. " $\mathrm{P}<0.05$ vs. sham group; ${ }^{\mathrm{P}}<0.05$ vs. HF group. IMD, intermedin; HF, heart failure.

quadrants at a x400 magnification and one microscopic field with the highest nerve density in each quadrant was selected for nerve counting. The mean nerve density of four selected fields was used to represent the nerve density of that slide.

Western blotting. Briefly, heart tissues were minced and then lysed for $20 \mathrm{~min}$ on ice with lysis buffer (Beyotime Institute of Biotechnology, Haimen, China). The lysates were centrifuged at $10,000 \mathrm{x} \mathrm{g}$ for $15 \mathrm{~min}$ at $4^{\circ} \mathrm{C}$ and the supernatants were collected. Total protein was extracted, the concentrations were determined using a bicinchoninic acid assay kit (Beyotime Institute of Biotechnology, Haimen, China). Equal amounts of proteins $(20 \mu \mathrm{g})$ were separated by SDS-PAGE (10\% separating gel and 5\% stacking gel; $80 \mathrm{~V} ; 120 \mathrm{~min}$ ) and subsequently transferred onto PVDF membranes (EMD Millipore, Billerica, MA, USA). After that, the membranes were blocked with $5 \%$ non-fat milk for $2 \mathrm{~h}$ at room temperature. Membranes were then incubated with rabbit monoclonal antibodies against TH $(1: 2,000)$ and GAP43 $(1: 10,000)$, a rabbit polyclonal antibody against NGF (1:2,000, cat no. ab6199; Abcam), and a mouse monoclonal antibody against GAPDH (1:5,000, cat no. 60004-1-Ig; ProteinTech Group, Inc., Chicago, IL, USA) overnight at $4^{\circ} \mathrm{C}$. The membranes were then washed and incubated with HRP-conjugated goat anti-rabbit (1:5,000, 111-035-003; Jackson ImmunoResearch Laboratories, Inc., West Grove, PA, USA) and anti-mouse IgG secondary antibodies (1:2,000, cat no. A0216; Beyotime Institute of Biotechnology) at room temperature for $1 \mathrm{~h}$. The bands were visualized using an Enhanced Chemiluminescence kit (EMD Millipore). The protein expressions were normalized to GAPDH protein content.

Measurement of plasma B-type natriuretic peptide (BNP) level. On the day of sacrifice, $1 \mathrm{ml}$ fresh blood was collected from all rats via the inferior vena cava and centrifuged at $1,500 \mathrm{x} g$ for $15 \mathrm{~min}$ at $4^{\circ} \mathrm{C}$. Then supernatants were collected and stored at $-80^{\circ} \mathrm{C}$. Plasma BNP levels were determined using an enzyme-linked immunosorbent assay (ELISA) kit (cat no. WEA541Ra; Wuhan USCN Business Co., Ltd., Wuhan, China).

Detection of malondialdehyde (MDA) level and superoxide dismutase (SOD) activity. Prior to the detection of the MDA levels and SOD activity the protein concentration was quantified using a bicinchoninic acid assay kit (Beyotime Institute of Biotechnology). Then the remaining ventricle samples were homogenized in saline and centrifuged at $1,000 \mathrm{xg}$ for $15 \mathrm{~min}$ at $4^{\circ} \mathrm{C}$. The supernatants were collected and the level of MDA and the activity of SOD were detected using commercial kits (Nanjing Jiancheng Bioengineering Research Institute, Nanjing, China) according to the manufacturer's protocol. The level of MDA is expressed as nmol/mgprot and the activity of SOD is expressed as U/mgprot.

Statistical analyses. Data are presented as the mean \pm standard deviation and data analysis was performed by SPSS 19.0 software (IBM SPSS, Armonk, NY, USA). Statistical differences between groups were analyzed using one-way analysis of variance followed by the Student's Newman-Keuls post-hoc test after normality distribution was evaluated. $\mathrm{P}<0.05$ was considered to indicate a statistically significant difference.

\section{Results}

Effects of IMD on cardiac function. Representative echocardiograms are presented in Fig. 1A. Compared with the HF group, IMD treatment significantly increased LVEF (32.5 \pm 4.0 vs. $27.8 \pm 5.1 \%$; $\mathrm{P}<0.05$; Fig. $1 \mathrm{~B})$ and LVFS $(15.8 \pm 2.2$ vs. $13.6 \pm 2.6 \%$; $\mathrm{P}<0.05$; Fig. $1 \mathrm{C})$. A significant increase in \pm LVdp/dtmax and a decrease in LVEDP were observed in IMD treatment group in comparison with the HF group (Table I). In addition, IMD treatment significantly decreased arterial 

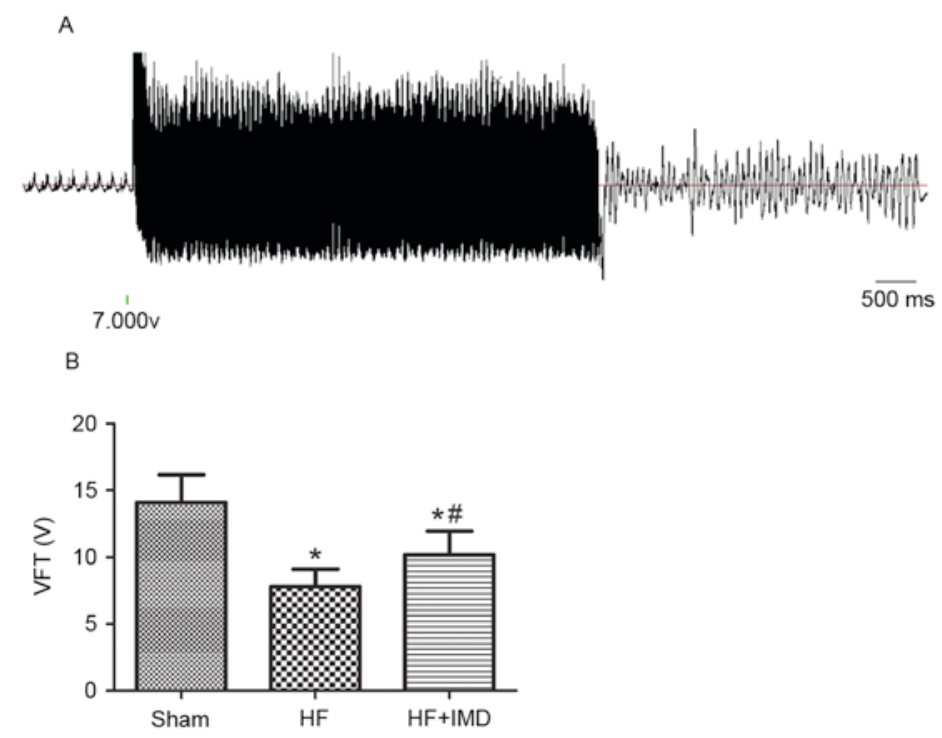

Figure 2. IMD increases VFT in rats with post-myocardial infarction HF. (A) A representative ventricle fibrillation induced by ventricle electric stimulation in an HF rat treated with saline-the VFT of this rat was 7 V. (B) Quantification of VFT among the three groups (B). Data are presented as the mean \pm standard deviation. ${ }^{*} \mathrm{P}<0.05$ vs. sham group; ${ }^{*} \mathrm{P}<0.05$ vs. HF group. HF, heart failure; VFT, ventricular fibrillation threshold; IMD, intermedin.

Table I. Body weight and haemodynamics at the end of the study.

\begin{tabular}{lccc}
\hline & Sham & HF & HF+IMD \\
\hline BW (g) & $384 \pm 8$ & $384 \pm 11$ & $389 \pm 13$ \\
HR (bpm) & $371 \pm 8$ & $373 \pm 9$ & $373 \pm 7$ \\
SBP (mmHg) & $133 \pm 11$ & $134 \pm 9$ & $112 \pm 9^{\mathrm{a}, \mathrm{b}}$ \\
DBP (mmHg) & $89 \pm 5$ & $85 \pm 6$ & $66 \pm 7^{\mathrm{a}, \mathrm{b}}$ \\
LVEDP (mmHg) & $5 \pm 2$ & $20 \pm 4^{\mathrm{a}}$ & $11 \pm 2^{\mathrm{a}, \mathrm{b}}$ \\
+ LVdp/dtmax & $7721 \pm 553$ & $3270 \pm 544^{\mathrm{a}}$ & $4469 \pm 39^{\mathrm{a}, \mathrm{b}}$ \\
$(\mathrm{mmHg} / \mathrm{s})$ & & & \\
$-\mathrm{LVdp} / \mathrm{dtmax}$ & $5971 \pm 443$ & $2959 \pm 507^{\mathrm{a}}$ & $3908 \pm 326^{\mathrm{a}, \mathrm{b}}$ \\
$(\mathrm{mmHg} / \mathrm{s})$ & & & \\
\hline
\end{tabular}

Data are presented as the mean \pm standard deviation. ${ }^{\mathrm{a}} \mathrm{P}<0.05 \mathrm{vs}$. sham group; ${ }^{\text {} P}<0.05$ vs. HF group. BW, body weight; $\mathrm{LV}$, left ventricle; HR, heart rate; SBP, systolic blood pressure; DBP, diastolic blood pressure; LVEDP, left ventricular end-diastolic pressure; $\pm \mathrm{LVdp} / \mathrm{dt}_{\max }$, the maximal rate of left ventricular pressure increase and decrease; HF, heart failure; IMD, intermedin.

blood pressure compared with the sham group and the HF group (Table I).

Effects of IMD on VFT. A representative ventricle fibrillation induced by ventricle electric stimulation in an HF rat treated with saline is presented in Fig. 1A. The VFT of the HF group was significantly reduced compared with the sham group (7.8 \pm 1.3 vs. $14.1 \pm 2.1 \mathrm{~V}$; $\mathrm{P}<0.05$; Fig. $2 \mathrm{~B}$ ). IMD induced a rise of VFT compared with the HF group (10.2 \pm 1.8 vs. $7.8 \pm 1.3 \mathrm{~V}$; $\mathrm{P}<0.05$; Fig. 2B).

Effects of IMD on cardiac morphology. There were no significant differences in body weight between the three groups at the end of experiment (Table I). However, the left ventricle weight/body weight (LVW/BW) value was significantly increased in the HF group compared with the sham group ( $2.85 \pm 0.08$ vs. $2.48 \pm 0.05$; $\mathrm{P}<0.05$; Fig. $3 \mathrm{~A}$ and $\mathrm{B})$. A significant decrease in LVW/BW was observed in the IMD treatment group compared with the HF group $(2.76 \pm 0.09$ vs. $2.85 \pm 0.08 ; \mathrm{P}<0.05 ;$ Fig. 3B). However, the infarct size between the HF group and IMD treatment group was not significantly different (Fig. 3C).

Effects of IMD on TH- and GAP43-positive nerve fiber density. Representative immunostaining for $\mathrm{TH}-$ and GAP43-positive nerve fibers in the peri-infarct zone are presented in Fig. 4A. The densities of TH- and GAP43-positive nerve fibers in the peri-infact zone of the HF group were significantly increased compared with the sham group (TH, $9534 \pm 1150$ vs. $2509 \pm 281 \mu \mathrm{m}^{2} / \mathrm{mm}^{2}$; GAP $43,11367 \pm 1375$ vs. $5073 \pm 854 \mu \mathrm{m}^{2} / \mathrm{mm}^{2}$; all $\mathrm{P}<0.05$; Fig. $4 \mathrm{~B}$ and $\mathrm{C}$, respectively). However, a significant decrease in the densities of TH- and GAP43-positive nerve fibers was observed in the IMD treatment group compared with the HF group (TH, $7532 \pm 825$ vs. $9534 \pm 1150 \mu \mathrm{m}^{2} / \mathrm{mm}^{2}$; GAP43, $8554 \pm 615$ vs. $11367 \pm 1375 \mu \mathrm{m}^{2} / \mathrm{mm}^{2}$; all $\mathrm{P}<0.05$; Fig. 4B and C).

Effects of IMD on the protein expression levels of TH, GAP43 and NGF. Representative western blot images are presented in Fig. 5A. The protein expression levels of TH, GAP43 and NGF were significantly increased in the HF group compared with the sham group (TH, $0.710 \pm 0.053$ vs. $0.419 \pm 0.034$; GAP43, $0.422 \pm 0.018$ vs. $0.197 \pm 0.010$ and NGF: $0.439 \pm 0.013$ vs. $0.213 \pm 0.013$; all $\mathrm{P}<0.05$; Fig. $5 \mathrm{~B}-\mathrm{D}$, respectively). IMD treatment significantly decreased the protein expression levels of TH, GAP43 and NGF compared with the HF group (TH, $0.530 \pm 0.038$ vs. $0.710 \pm 0.053$; GAP43, $0.320 \pm 0.021$ vs. $0.422 \pm 0.018$ and NGF, $0.270 \pm 0.021$ vs. $0.439 \pm 0.013$; all $\mathrm{P}<0.05$; Fig. 5B-D, respectively). 

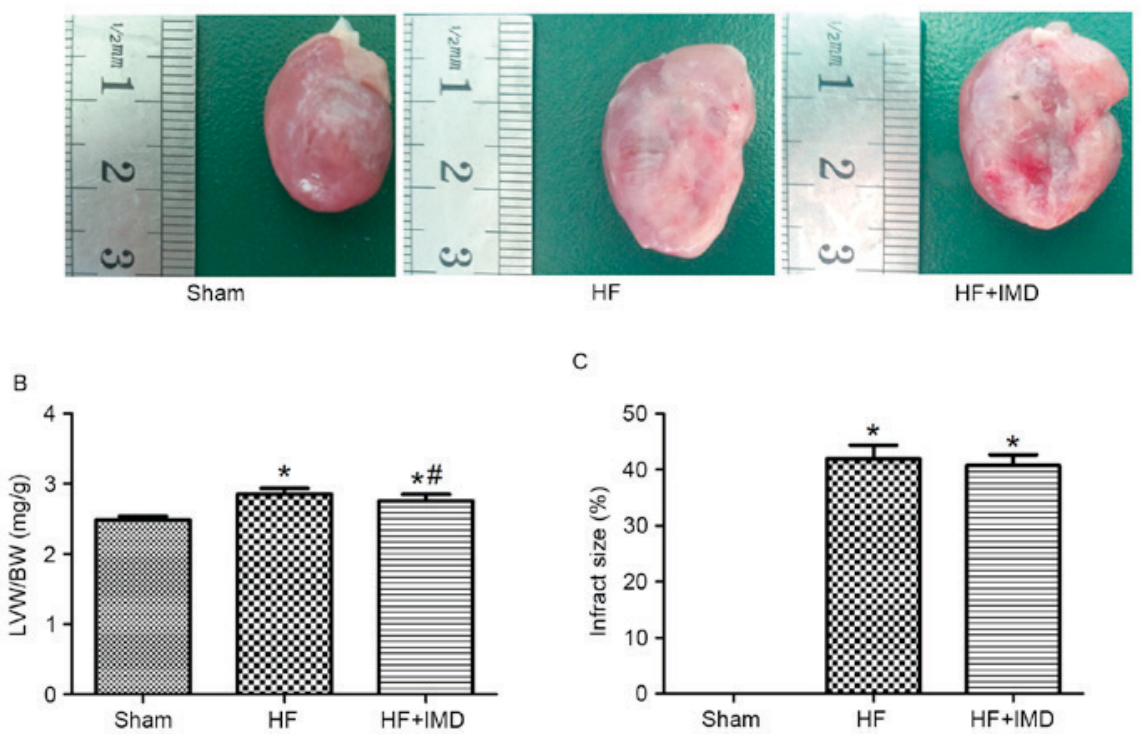

C

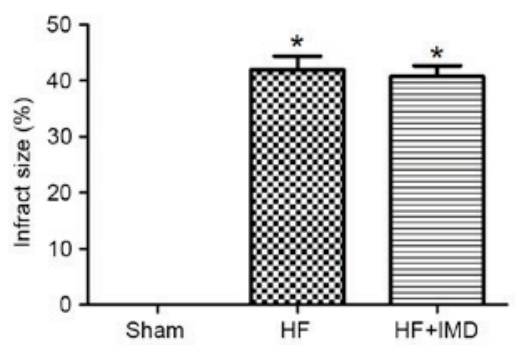

Figure 3. IMD suppresses cardiac hypertrophy in rats with post-myocardial infarction HF. (A) Representative images of hearts from the sham, HF and $\mathrm{HF}+\mathrm{IMD}$ groups. Quantification of (B) the LVW/BW ratio and (C) infarct size in the three groups. Data are presented as the mean \pm standard deviation. ${ }^{*} \mathrm{P}<0.05$ vs. sham group; ${ }^{\text {P }}<0.05$ vs. HF group. LVW, left ventricle weight; BW, body weight; IMD, intermedin; HF, heart failure.

A
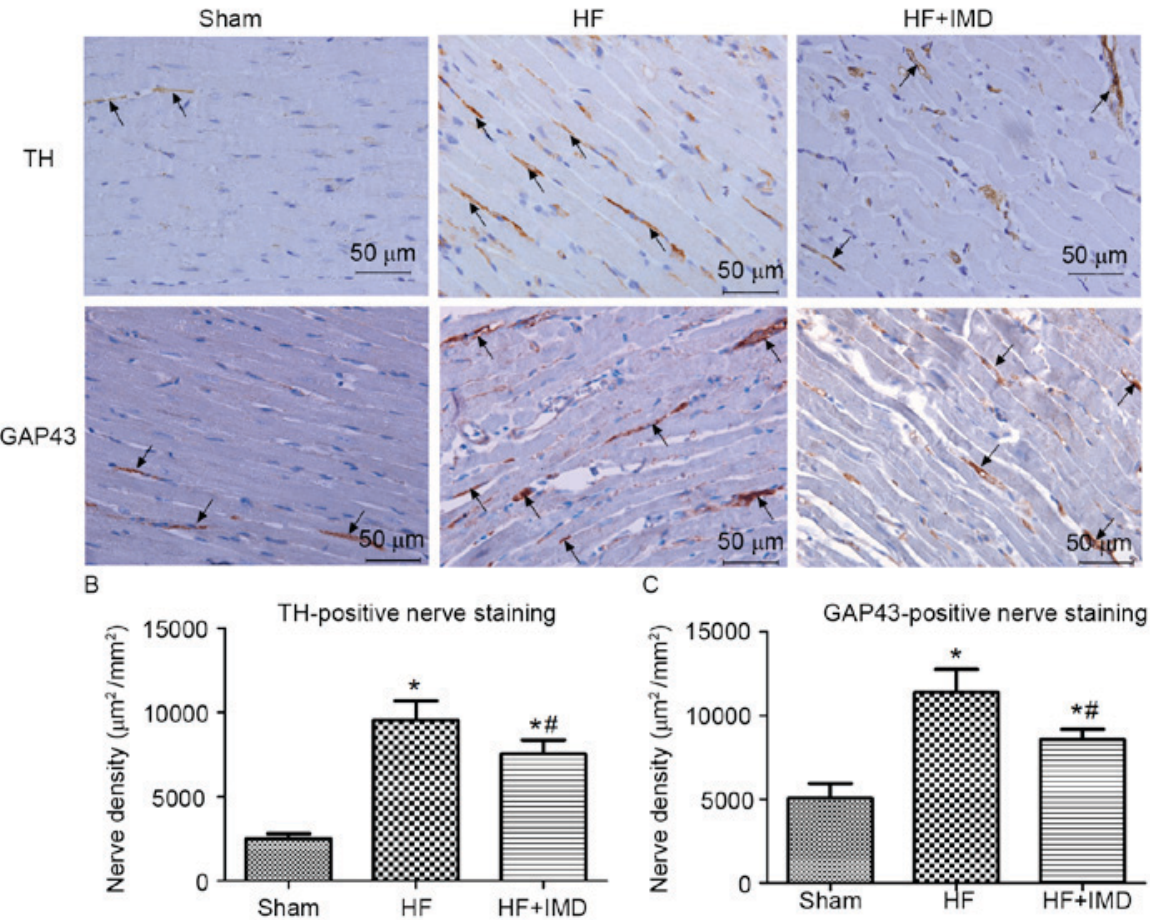

Figure 4. Effect of IMD on sympathetic neural remodeling following myocardial infarction. (A) Representative immunostaining for TH- and GAP43-positive nerve fibers in the peri-infarct zone (magnification, x400; scale bar=50 $\mu \mathrm{m}$ ). Quantification of (B) TH- and (C) GAP43-positive nerve fiber densities. Data are presented as the mean \pm standard deviation. ${ }^{*} \mathrm{P}<0.05$ vs. sham group; ${ }^{*} \mathrm{P}<0.05$ vs. HF group. TH, tyrosine hydroxylase; GAP43, growth associated protein 43 ; IMD, intermedin; HF, heart failure.

Effects of IMD on plasma BNP levels and oxidative stress in the heart. The plasma BNP level in the HF group was significantly increased compared with the sham group. IMD treatment significantly decreased the plasma BNP level compared with the HF group (Table II). The MDA level in the peri-infarct zone of the HF group was significantly increased, while the SOD activity was significantly decreased compared with the sham group. IMD significantly decreased the MDA level and increased SOD activity in comparison to the HF group (Table II).

\section{Discussion}

The present study demonstrated that long-term treatment with IMD improves cardiac function and attenuates sympathetic neural remodeling in a rat model of post-MI HF. Furthermore, 
A

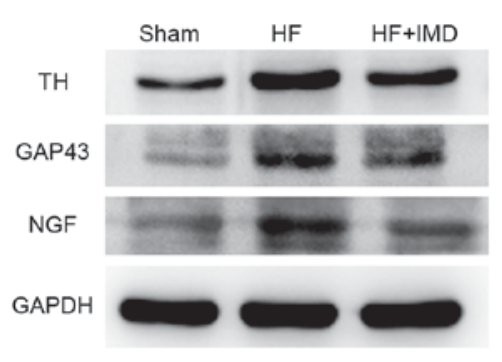

C

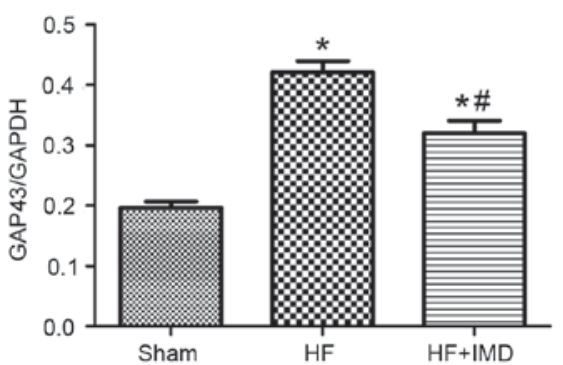

B

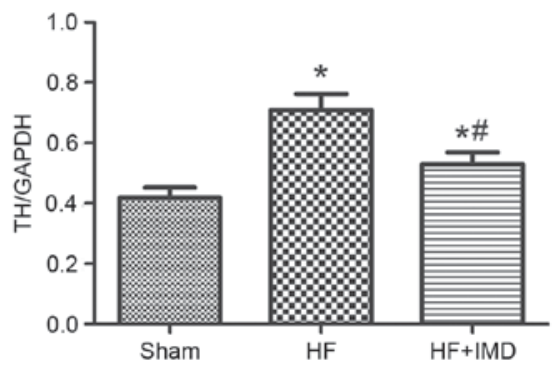

D

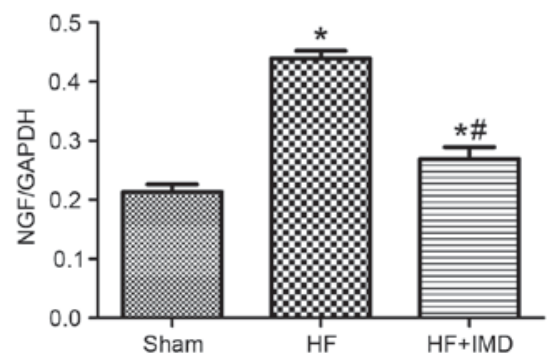

Figure 5. Effect of IMD on protein expression levels of TH, GAP43 and NGF following myocardial infarction. (A) Representative western blot images and quantification of (B) TH, (C) GAP43 and (D) NGF protein expression levels in the peri-infarct zone. GAPDH served as an internal control. Data are presented as the mean \pm standard deviation. ${ }^{*} \mathrm{P}<0.05$ vs. sham group; ${ }^{*} \mathrm{P}<0.05$ vs. HF group. $\mathrm{TH}$, tyrosine hydroxylase; GAP43, growth associated protein 43 ; IMD, intermedin; $\mathrm{HF}$, heart failure; NGF, nerve growth factor.

Table II. Plasma BNP level, MDA content and SOD activity in the myocardium.

\begin{tabular}{lccc}
\hline & Sham & HF & HF+IMD \\
\hline BNP (pg/ml) & $154.16 \pm 33.34$ & $1089.75 \pm 124.22^{\mathrm{a}}$ & $784.51 \pm 190.81^{\mathrm{a}, \mathrm{b}}$ \\
MDA (nmol/mgprot) & $0.98 \pm 0.25$ & $1.47 \pm 0.09^{\mathrm{a}}$ & $1.29 \pm 0.18^{\mathrm{a}, \mathrm{b}}$ \\
SOD (U/mgprot) & $404.10 \pm 21.95$ & $296.37 \pm 60.90^{\mathrm{a}}$ & $354.66 \pm 16.96^{\mathrm{a}, \mathrm{b}}$ \\
\hline
\end{tabular}

Data are presented as the mean \pm standard deviation. ${ }^{\mathrm{a}} \mathrm{P}<0.05$ vs. sham group; ${ }^{\mathrm{b}} \mathrm{P}<0.05$ vs. HF group. BNP, B-type natriuretic peptide; MDA, malondialdehyde; SOD, superoxide dismutase; HF, heart failure; IMD, intermedin.

the present study revealed that IMD significantly increased the VFT in ischemic rat hearts, which may reduce the incidence of ventricular arrhythmia. Notably, the effect of IMD on sympathetic innervation was associated with its anti-oxidative property.

As a member of CGRP family, IMD had hypotensive and positive inotropic effects via activation of the CRLR/RAMP complexes (21-23). These findings suggested that IMD may be beneficial in treating HF. Intravenous infusion of IMD to HF sheep for 90 min decreased arterial blood pressure and enhanced cardiac contractility and output (13). The BNP level in the IMD treatment group was decreased in comparison with the $\mathrm{HF}$ group at a low dose $(0.6 \mu \mathrm{g} / \mathrm{kg} / \mathrm{h})$; however, the difference did not reach statistical significance (13). The present study observed that long-term administration of IMD significantly decreased the arterial blood pressure, enhanced cardiac contractility, and elevated LVEF and LVFS. Furthermore, it was demonstrated that low dose $(0.6 \mu \mathrm{g} / \mathrm{kg} / \mathrm{h})$ IMD decreases the BNP level, which mirrored the improvement of cardiac function.
Angiotensin II (Ang II) is hypothesized to serve a major role in ventricle remodeling in heart failure, which promotes myocardium hypertrophy (24). Blockade of the renin-angiotensin system attenuates cardiomyocyte hypertrophy in post-MI HF (18). It has been reported that the cell size of H9c2 cells was increased by Ang II treatment, while IMD significantly attenuates hypertrophy in these cells (25). The underlying mechanism involved its activation of both cyclic adenosine monophosphate/protein kinase A and mitogen-activated protein kinase/extracellular signal-regulated kinase 1/2 (MAPK/ERK1/2) signaling pathways, which further activated autophagy in hypertrophic cardiomyocytes (25). The present study established an ischemia-induced HF rat model and observed that long-term administration of IMD prevented cardiac hypertrophy, as assessed by cardiac morphology measurements and echocardiography. Therefore, IMD may ameliorate cardiac hypertrophy, at least in part, via abolishing the adverse effects of Ang II in ischemic rat hearts. However, the more specific mechanism and the downstream signaling pathway between IMD and Ang II requires further 
investigation. In respect to infarct size, there was no significant difference between the HF and IMD treatment groups in this study.

To the best of our knowledge, the effects of IMD on sympathetic innervation after MI have not previously been studied. The present study demonstrated that long-term treatment with IMD suppresses sympathetic neural remodeling and increased the VFT after MI.

$\mathrm{TH}$ is a rate-limiting enzyme of norepinephrine, which reflects the activity of sympathetic nerves (26). GAP43, which is expressed at the nerve growth cones, is a marker of new sprouting axons (26). Sympathetic neural remodeling following MI was observed in MI patients and animal models, which exhibited increased densities of TH- and GAP43-positive nerve fibers $(5,27)$. Subsequent studies demonstrated that the new sprouting nerves were more obvious in the peri-infarct zone $(28,29)$. In accordance with a previous study, the present study revealed that the densities of TH- and GAP43-positive nerve fibers were significantly increased in the peri-infarct zone, together with an increase in protein expression levels of TH and GAP43. Compared with the HF group, treatment with IMD decreased sympathetic nerve density and downregulated the protein expression levels of TH and GAP43.

It has been demonstrated that increased sympathetic nerve density accounts for the incidence of ventricular arrhythmia and SCD in patients with severe HF (27). Furthermore, catheter-based mapping techniques confirmed that the original site of most ventricular arrhythmia is the peri-infarct zone in patients with MI (30). Therefore, to investigate whether IMD could reduce the incidence of ventricular arrhythmia, the present study determined the VFT, an indicator to evaluate the incidence of ventricular arrhythmia. It was observed that IMD treatment increased the VFT compared with the HF group, which suggested that IMD could decrease the incidence of ventricular arrhythmia.

The underlying mechanisms of sympathetic hyperinnervation following MI remain to be clearly elucidated. It is generally agreed that NGF, which is essential for sympathetic nerve growth and survival (31), is involved in the neural regeneration process. A previous study demonstrated that the expression of NGF was increased in the peri-infarct zone (29). Furthermore, infusion of NGF to the left stellate ganglion elevated the densities of TH- and GAP-43 positive nerve fibers and increased the incidence of SCD in MI dogs (5). Similarly, overexpression of NGF in transgenic mouse heart resulted in sympathetic hyperinnervation (32).

Oxidative stress is characterized by increased oxidant production and impaired anti-oxidant defenses. It has been demonstrated that oxidative stress is increased and has a critical role in sympathetic neural remodeling following MI (6). A further study revealed that oxidative stress augmented the expression of NGF in infarcted rat hearts (6). Inhibiting the generation of superoxide decreased the expression of NGF and attenuated sympathetic neural remodeling following MI (7-9). IMD exerted an anti-oxidative effect via activating the MAPK/ERK1/2 pathway, which protected the heart from ischemia/reperfusion injury $(14,33)$. The present study observed that IMD increased the activity of SOD, decreased the level of MDA and downregulated the expression of NGF in the peri-infarct zone, which suggested that IMD attenuates sympathetic neural remodeling via inhibiting oxidative stress.

In conclusion, the present study demonstrated that with favorable haemodynamic and anti-oxidative effects, long-term administration of IMD improves cardiac function, prevents sympathetic neural remodeling and reduces the incidence of ventricular arrhythmia in rats with post-MI HF. These results implicate IMD as a potential therapeutic agent for the treatment of HF.

\section{Acknowledgements}

The present study was partially supported by the National Nature Science Foundation of China (grant no. 30971265) and the Shanghai Rising-Star Program (grant no. 08QA1404100).

\section{References}

1. Felker GM, Benza RL, Chandler AB, Leimberger JD, Cuffe MS, Califf RM and Gheorghiade M; OPTIME-CHF Investigators: Heart failure etiology and response to milrinone in decompensated heart failure: Results from the OPTIME-CHF study. J Am Coll Cardiol 41: 997-1003, 2003.

2. Kurrelmeyer K, Kalra D, Bozkurt B, Wang F, Dibbs Z, Seta Y, Baumgarten G, Engle D, Sivasubramanian N and Mann DL: Cardiac remodeling as a consequence and cause of progressive heart failure. Clin Cardiol 21 (12 Suppl 1): I14-I19, 1998.

3. Colucci WS, Sawyer DB, Singh K and Communal C: Adrenergic overload and apoptosis in heart failure: Implications for therapy. J Card Fail 6 (2 Suppl 1): S1-S7, 2000.

4. Swissa M, Zhou S, Gonzalez-Gomez I, Chang CM, Lai AC, Cates AW, Fishbein MC, Karagueuzian HS, Chen PS and Chen LS: Long-term subthreshold electrical stimulation of the left stellate ganglion and a canine model of sudden cardiac death. J Am Coll Cardiol 43: 858-864, 2004.

5. Cao JM, Chen LS, KenKnight BH, Ohara T, Lee MH, Tsai J, Lai WW, Karagueuzian HS, Wolf PL, Fishbein MC and Chen PS: Nerve sprouting and sudden cardiac death. Circ Res 86: 816-821, 2000.

6. Lee TM, Chen CC and Hsu YJ: Differential effects of NADPH oxidase and xanthine oxidase inhibition on sympathetic reinnervation in postinfarct rat hearts. Free Radic Biol Med 50: 1461-1470, 2011.

7. Lee TM, Chen WT, Yang CC, Lin SZ and Chang NC: Sitagliptin attenuates sympathetic innervation via modulating reactive oxygen species and interstitial adenosine in infarcted rat hearts. J Cell Mol Med 19: 418-429, 2015.

8. Lee TM, Lin SZ and Chang NC: Antiarrhythmic effect of lithium in rats after myocardial infarction by activation of $\mathrm{Nrf} 2 / \mathrm{HO}-1$ signaling. Free Radic Biol Med 77: 71-81, 2014.

9. Xin P, Pan Y, Zhu W, Huang S, Wei M and Chen C: Favorable effects of resveratrol on sympathetic neural remodeling in rats following myocardial infarction. Eur J Pharmacol 649: 293-300, 2010.

10. Roh J, Chang CL, Bhalla A, Klein C and Hsu SY: Intermedin is a calcitonin/calcitonin gene-related peptide family peptide acting through the calcitonin receptor-like receptor/receptor activity-modifying protein receptor complexes. J Biol Chem 279: 7264-7274, 2004.

11. Bell D and McDermott BJ: Intermedin (adrenomedullin-2): A novel counter-regulatory peptide in the cardiovascular and renal systems. Br J Pharmacol 153 (Suppl 1): S247-S262, 2008.

12. Hirose T, Totsune K, Mori N, Morimoto R, Hashimoto M, Nakashige Y, Metoki H, Asayama K, Kikuya M, Ohkubo T, et al: Increased expression of adrenomedullin 2/intermedin in rat hearts with congestive heart failure. Eur J Heart Fail 10: 840-849, 2008.

13. Rademaker MT, Charles CJ, Nicholls MG and Richards AM: Hemodynamic, hormonal and renal actions of adrenomedullin 2 in experimental heart failure. Circ Heart Fail 1: 134-142, 2008.

14. Zhao L, Peng DQ, Zhang J, Song JQ, Teng X, Yu YR, Tang CS and Qi YF: Extracellular signal-regulated kinase 1/2 activation is involved in intermedin1-53 attenuating myocardial oxidative stress injury induced by ischemia/reperfusion. Peptides 33: 329-335, 2012. 
15. Li H, Bian Y, Zhang N, Guo J, Wang C, Lau WB and Xiao C: Intermedin protects against myocardial ischemia-reperfusion injury in diabetic rats. Cardiovasc Diabetol 12: 91, 2013.

16. Qiao X, Li RS, Li H, Zhu GZ, Huang XG, Shao S and Bai B: Intermedin protects against renal ischemia-reperfusion injury by inhibition of oxidative stress. Am J Physiol Renal Physiol 304: F112-F119, 2013.

17. Hong Y, Hay DL, Quirion R and Poyner DR: The pharmacology of adrenomedullin 2/intermedin. Br J Pharmacol 166: 110-120, 2012.

18. Connelly KA, Advani A, Advani S, Zhang Y, Thai K, Thomas S, Krum H, Kelly DJ and Gilbert RE: Combination angiotensin converting enzyme and direct renin inhibition in heart failure following experimental myocardial infarction. Cardiovasc Ther 31: 84-91, 2013.

19. Vracko R, Thorning D and Frederickson RG: Nerve fibers in human myocardial scars. Hum Pathol 22: 138-146, 1991.

20. Pfeffer MA and Braunwald E: Ventricular remodeling after myocardial infarction. Experimental observations and clinical implications. Circulation 81: 1161-1172, 1990.

21. Hagner S, Stahl U, Knoblauch B, McGregor GP and Lang RE: Calcitonin receptor-like receptor: Identification and distribution in human peripheral tissues. Cell Tissue Res 310: 41-50, 2002.

22. Dong F, Taylor MM, Samson WK and Ren J: Intermedin (adrenomedullin-2) enhances cardiac contractile function via a protein kinase $\mathrm{C}$ - and protein kinase A-dependent pathway in murine ventricular myocytes. J Appl Physiol (1985) 101: 778-784, 2006.

23. Fujisawa Y, Nagai Y, Miyatake A, Miura K, Nishiyama A, Kimura $\mathrm{S}$ and Abe Y: Effects of adrenomedullin 2 on regional hemodynamics in conscious rats. Eur J Pharmacol 558: 128-132, 2007.

24. Sun Y, Zhang J, Zhang JQ and Weber KT: Renin expression at sites of repair in the infarcted rat heart. J Mol Cell Cardiol 33: 995-1003, 2001.

25. Chen H, Wang X, Tong M, Wu D, Wu S, Chen J, Wang X, Wang X, Kang Y, Tang H, et al: Intermedin suppresses pressure overload cardiac hypertrophy through activation of autophagy. PLoS One 8: e64757, 2013.

26. Chen PS, Chen LS, Cao JM, Sharifi B, Karagueuzian HS and Fishbein MC: Sympathetic nerve sprouting, electrical remodeling and the mechanisms of sudden cardiac death. Cardiovasc Res 50: 409-416, 2001.
27. Cao JM, Fishbein MC, Han JB, Lai WW, Lai AC, Wu TJ, Czer L, Wolf PL, Denton TA, Shintaku IP, et al: Relationship between regional cardiac hyperinnervation and ventricular arrhythmia. Circulation 101: 1960-1969, 2000.

28. Li W, Knowlton D, Van Winkle DM and Habecker BA: Infarction alters both the distribution and noradrenergic properties of cardiac sympathetic neurons. Am J Physiol Heart Circ Physiol 286: H2229-H2236, 2004.

29. Hasan W, Jama A, Donohue T, Wernli G, Onyszchuk G, Al-Hafez B, Bilgen M and Smith PG: Sympathetic hyperinnervation and inflammatory cell NGF synthesis following myocardial infarction in rats. Brain Res 1124: 142-154, 2006.

30. Verma A, Marrouche NF, Schweikert RA, Saliba W, Wazni O, Cummings J, Abdul-Karim A, Bhargava M, Burkhardt JD, Kilicaslan F, et al: Relationship between successful ablation sites and the scar border zone defined by substrate mapping for ventricular tachycardia post-myocardial infarction. J Cardiovasc Electrophysiol 16: 465-471, 2005.

31. Lindsay RM, Thoenen H and Barde YA: Placode and neural crest-derived sensory neurons are responsive at early developmental stages to brain-derived neurotrophic factor. Dev Biol 112: 319-328, 1985.

32. Hassankhani A, Steinhelper ME, Soonpaa MH, Katz EB Taylor DA, Andrade-Rozental A, Factor SM, Steinberg JJ, Field LJ and Federoff HJ: Overexpression of NGF within the heart of transgenic mice causes hyperinnervation, cardiac enlargement and hyperplasia of ectopic cells. Dev Biol 169: 309-321, 1995.

33. Wang J, Shen YH, Utama B, Wang J, LeMaire SA, Coselli JS, Vercellotti GM and Wang XL: HCMV infection attenuates hydrogen peroxide induced endothelial apoptosis-involvement of ERK pathway. FEBS Lett 580: 2779-2787, 2006. 